\title{
Essais
}

Revue interdisciplinaire d'Humanités

\section{La désymbolisation du cimetière contemporain}

\section{Patrick Baudry}

\section{OpenEdition}

Journals

Édition électronique

URL : http://journals.openedition.org/essais/5817

DOI : 10.4000/essais. 5817

ISSN : 2276-0970

\section{Éditeur}

École doctorale Montaigne Humanités

\section{Édition imprimée}

Date de publication : 15 janvier 2015

Pagination : 126-138

ISBN : 978-2-9544269-4-5

ISSN : 2417-4211

\section{Référence électronique}

Patrick Baudry, "La désymbolisation du cimetière contemporain », Essais [En ligne], 6 | 2015, mis en ligne le 30 novembre 2020, consulté le 02 décembre 2020. URL : http://journals.openedition.org/ essais/5817 ; DOI : https://doi.org/10.4000/essais.5817 


\section{La désymbolisation du cimetière contemporain}

\section{Patrick Baudry}

\section{La mort inutile}

La ville contemporaine s'organise contre l'idée de mort, elle prétend ignorer le mourir et elle défait les articulations qui pouvaient se nouer entre les vivants et les morts. L'espace urbanisé suppose la promotion de valeurs en rapport desquelles la mort devient une anti-valeur. L'énergie perpétuelle, la compétitivité, la rentabilité, la productivité, ou encore la rationalisation si ce n'est la rationalité, s'opposent en force à la non-rationalité de la mort, à l'inutilité qu'elle signifie et à l'arrêt auquel elle oblige. Le mort est un improductif, il n'entre pas dans le circuit du profit. La mort est une absurdité qui porte à relativiser les projets, les ambitions, les appétits de puissance ou les volontés de pouvoir : à moins que, mise au service de ces volontés, elle ne signifie le saccage de la planète, la domination du faible, l'exploitation de la créativité au seul service de la reproduction d'un système qui vit de sa propre perpétuation. Quant au mourir ${ }^{1}$, il suppose non pas seulement l'agonie dont on ne verrait plus aujourd'hui l'enjeu - tout se passant comme s'il fallait pouvoir en finir avant que d'être mort $^{2}-$, mais la temporalité et donc la relation à autrui.

La question de la mort - ce n'est pas qu'un problème ou une affaire d'individu - est bien entendu la question de la vie, des priorités qu'on porte dans l'existence et du sens qu'on veut lui donner. On comprend donc que LouisVincent Thomas ait pu parler "d'anthropo-thanatologie polémique " ${ }^{3}$. Il ne saurait s'agir d'aller seulement s'émouvoir de quelques changements de " représentations ", comme si des "vues " avaient changé et comme s'il suffisait de faire l'histoire de diapositives mentales qui se seraient succédées de manière linéaire. L'individualisation du rapport à la mort dont on veut souligner la

\footnotetext{
Voir Patrick Baudry, Pourquoi des soins palliatifs ?, Cirey, Éditions Châtelet-Voltaire, 2013.

Voir Patrick Baudry, Le Corps extrême, Paris, L’Harmattan, 1991.

Voir Louis-Vincent Thomas, Mort et pouvoir (1978), Paris, Payot, 1999.
} 
signification positive en parlant d'une "personnalisation " des funérailles - quelques interventions de proches du défunt dans le déroulement professionnalisé des obsèques - ou qui s'affirme dans le choix que l'on fait de son vivant de sa propre cérémonie funéraire, signifie la perte de solidarités collectives, l'abandon du mort à son cercueil et celui des endeuillés à leur chagrin " intime ». On a sans doute du mal à imaginer qu'un quartier de Paris ait pu entrer en manifestation (au $\mathrm{XV}^{\mathrm{e}}$ siècle) pour détourner un convoi funéraire et l'obliger à se diriger vers le cimetière où se trouvaient " ses morts ". Ou encore la précaution policière qu'il fallut prendre pour fermer le cimetière des Saints Innocents par crainte des réactions sociales ${ }^{4}$. À présent, l'endroit où sont mis les morts compte sans doute pour chacun, mais laisse à peu près indifférent son entourage non familial. Il convient actuellement de cacher sa peine à son environnement professionnel, tout comme de s'adonner à des funérailles discrètes. Le corbillard motorisé se mêle aujourd'hui à la circulation rapide (j'ai connu dans mon enfance le passage lent qu'il faisait dans nos rues en roulant au bord des trottoirs), et il n'est plus nécessairement noir, mais gris, bordeaux, bleu marine ou vert. Le vêtement du deuil disparait, de même que l'ensemble des signes sociaux au travers desquels le rapport collectif à la mort se visibilisait. Les étudiants d'aujourd'hui ne comprennent pas qu'un teinturier ait pu naguère installer dans sa vitrine un panonceau où l'on pouvait lire "Deuil en 24 heures ». Déjà le souci de la performance rapide apparaissait dans les années 1970, mais il s'agissait encore de composer avec des traditions a priori intangibles (devoir être vêtu de noir si l'on devait assister à des funérailles). Aujourd'hui le noir peut être la couleur de l'élégance, du chic ou du sexy, tandis que pour se rendre aux obsèques d'un proche il est recommandé de conserver ses habits quotidiens. C'est aussi bien le " macaron" de deuil au revers de la veste, ou le brassard noir qui auront progressivement disparu, comme les faire-part de décès (l'avis paru dans le journal en tient lieu), les processions funéraires ou les condoléances qu'on exprimait physiquement aux proches du défunt (un cahier où l'on marque son nom à l'entrée de l'église suffit à présent pour signaler qu’on était là).

\section{La disparition de la mort}

Longtemps la ville - ou la ville-village - contient les morts. Ou plutôt faut-il dire que l'espace des vivants suppose pour se déterminer son articulation à l'espace des morts. Ce monde est collectif: on n'y a pas sa tombe ou son caveau. L'institution de l'Église censée exercer une influence protectrice, la proximité de la tombe d'un saint ou d'un martyr qui pourraient intercéder auprès de Dieu, signifient, dans la pratique que l'on a de la terre et des lieux, qu'on y différencie

4 Voir Michel Ragon, L'espace et la mort, Paris, Albin Michel, 1981. 
cette symbolique articulatoire qui ne tient pas que de la juxtaposition. Les morts habitent la terre où les vivants existent en attendant le moment du " passage ". Puis la mort s'individualise. La tombe individuelle apparaît. L'hommage aux morts devient progressivement l'affaire d'une mémoire familiale. Autour du mourant qui n'est plus l'acteur principal d'un cérémonial de l'adieu, se tient un groupe de proches soucieux de la peine qui s'affirme de manière nouvelle parce qu'apparait la crainte de sa propre mort, la hantise de la fin de l'existence et le sentiment d'un arrêt de la vie qui signifie déjà une rupture. Les morts ne sont plus conjoints dans une vision de la vie, mais disjoints par le passage qui, à présent, les isolent. La mort devient, dans l'ordre médical qui en organise la gestion, une déconnexion. C'est pour des raisons d'hygiène et avec l'argument de respecter des morts étrangers que l'on édifie le cimetière moderne : loin des villes ou devant " ressembler à des villes vues de loin $»^{5}$. Le travail qui s'accomplit de la mémoire n'est plus identique : au monde communautaire des morts qui seraient ensemble se substitue le stockage des décédés. À la ville-village des morts qui vivraient " en dessous de la terre " (comme on le dit en Afrique Noire) s'oppose le rangement des morts « dans " une terre qui n'est plus l'espace d'une métamorphose ou d'une attente. Le fétichisme du caveau (pour reprendre l'expression de Louis-Vincent Thomas), le souci de passer l'aspirateur ${ }^{6}$ ou de frotter à la brosse la pierre tombale, signalent l'ambiguïté d'un rangement des défunts : à la fois ils n'y seraient plus (on le sait) et c'est pourtant là qu'il faudrait venir les « visiter».

Le lieu se réduit ici à l'emplacement : il s'agit bien d'installer géographiquement les morts, mais la gestion de l'espace l'emporte sur la qualification symbolique d'un destin ou d'une destination. Ou l'on peut dire qu'il s'agit bien de regrouper des cadavres, mais non plus de pratiquer un intervalle - un espace des morts -, c'est à dire un écart. Il s'agit sans doute d'espace des morts, au sens où de manière toute obligatoire on leur accorde une étendue, un morceau de terre ou un bout de terrain, mais non plus d'espacement avec ce que cela suppose de la mobilisation d'une culture dans un rapport aux défunts. Aujourd'hui où le tissu urbain s'est étendu, les cimetières, qu'on avait voulu placer en dehors des villes $^{7}$, se retrouvent compris dans le monde des vivants. Mais il faut souligner que le loyer baisse si la cuisine "donne " sur les caveaux et les mausolées, et que cette résidence silencieuse peut se côtoyer avec indifférence à moins qu'elle n'abrite quelques tombes de célébrités ou de stars (le cimetière se visite alors à la manière d'un musée) ou qu'elle ne possède de beaux arbres et de jolies pelouses (on pratique alors l'espace des morts comme un lieu de promenade).

\footnotetext{
5 Voir Michel Ragon, op. cit.

6 Voir Jean-Didier Urbain, La Société de conservation, Paris, Payot, 1978.

7 Voir Pascal Hintermeyer, Politiques de la mort, Paris, Payot, 1981.
} 
Pierre George m'avait dit un jour qu'une ville nouvelle d'Amérique du sud s'était mise à vivre à partir du premier enterrement. Dans nos sociétés, il n'est pas nécessaire que les planificateurs « oublient " le cimetière comme ce fut le cas pour la ville nouvelle du Vaudreuil. C'est toute l'idéologie du système urbain qui en fait l'impasse, comme si la mort n'appartenait de façon tautologique qu'aux morts, c'est-à-dire à un passé sans actualité, sans incidence sur nos vies.

La réduction de la personne au corps et la représentation très évidente de la mort comme cessation des activités vitales, peuvent générer dans le cadre des funérailles le choix de services qui permettent de gérer au mieux les impératifs du temps urbain : sans dépendre d'autorités extérieures dont la légitimité est réduite et dont les représentants (les prêtres n'en ont souvent plus le temps) sont absents au cimetière. Si $80 \%$ des obsèques sont aujourd'hui encore religieuses, la tendance est bien celle d'une professionnalisation du secteur funéraire : des spécialistes s'occupent des formalités administratives, du transport du corps, mais aussi et surtout de la cérémonie et éventuellement de l'assistance au deuil. L'assistant funéraire qui remplace l'ordonnateur des pompes funèbres, devient dans une société où le poids institutionnel de la religion s'amenuise, l'intermédiaire principal des familles. Il devient l'homme d'orchestre des opérations pratiques, mais il peut aussi - telle semble être l'ambition des entreprises - se poser comme l'organisateur du sens ${ }^{8}$ : un sens essentiellement réduit à la prise en charge des familles. La promotion de l'individu "libre " et le recul des institutions provoquent l'ouverture d'un marché. On ne s'étonne pas que le souci du confort de l'endeuillé réponde à la demande d'un public pour qui la mise en sens d'un rapport aux défunts tiendrait d'une préoccupation périmée. Ainsi les "Maisons funéraires " gérées comme des entreprises pourront-elles devenir les lieux de cultes de demain. Au lieu de multiplier des déplacements (du domicile ou de l'hôpital à l'église, puis de l'église au cimetière) «dommageables pour la rentabilité des matériels et du personnel " comme l'affirme un responsable d'entreprise funéraire ( $L e$ Monde, 1/11/2000), on pourra effectuer sur place la " cérémonie de l'adieu ».

Plus spectaculaire et plus significatif est sans doute le projet de création à Saint-Quentin-de-Baron dans la région de Bordeaux d'un complexe funéraire $\mathrm{du}$ " troisième millénaire » : "Le Refuge aux étoiles " (Sud-Ouest 2/04/2000). $\mathrm{Ce}$ « concept " aurait pu s'appliquer dans une quarantaine de sites en France. Il s'agissait d'installer sur un terrain d'une dizaine d'hectares un funérarium avec un hôtel-restaurant, des boutiques, un parc où l'on aurait pu se promener au son de musiques douces, de chants d'oiseaux et de bruits de cascades, ainsi qu'un "sublimatorium " dans lequel une ampoule, en un plafond vouté, aurait pu s'installer à chaque nouvelle crémation. Ce qui est intéressant dans

8 Voir Pascale Trompette et Olivier Boissin, «Entre les vivants et les morts : les pompes funèbres aux portes du marché ", Sociologie du travail, n 42, 2000. 
ce projet qui n’a jamais été réalisé, c’est le retour sous une forme adaptée à notre actualité du projet de cimetière régional tel que le Baron Haussmann en avait eu l'idée (elle aussi jamais concrétisée) : celle d'un cimetière à Mérysur-Oise relié à Paris par un chemin de fer spécialement conçu pour convoyer les morts et les vivants qui seraient venus leur rendre hommage. De façon régulière, peut-on dire, la question du cimetière se repose en Occident. Il s'agit de le fermer, de l'éloigner, de l'invisibiliser'. Il s'agirait aujourd'hui de l'absorber. Ici, le lieu des morts tiendraient de lieu pour les vivants : regroupement de familles dispersées, promenades, sensations de "grandeur du sacré » et shopping pourraient s'associer. Au lieu de se poser comme l'altérité même, la Figure des morts " apprivoisés " pourrait s'intégrer à la gestion optimale des spatialités et des temporalités urbaines. Il y a là sans doute le signe d'une absorption du territoire de la mort dans la régulation urbaine. Au lieu de s'opposer à la mort (voie coûteuse et sans issue), la ville urbanisée pourrait en faire un élément de son propre développement.

\section{L'individualité obligée}

La confrontation de la société devant la mort, l'adieu au mort, la construction de son destin, l'imagination de son statut, appartenaient à des institutions. C'est dans le registre de la religion que la souffrance et l'espoir s'exprimaient, que la séparation d'avec celui qui n'est plus et que le remaniement de la relation avec le défunt trouvaient leur sens. Aujourd'hui, on devrait constater l'appauvrissement de cette ritualité. Sans doute $80 \%$ des cérémonies funéraires se pratiquent encore dans le cadre d'une église. Mais il s'agirait presque de bénéficier des avantages d'un endroit où il faut que cela puisse se passer, bien plus que de passer par un lieu en se confrontant aux élaborations qu'il obligeait. Ainsi le "sacré " pourrait-il devenir bien plus sentimental et individuel qu'institutionnel et collectif. Une individualisation marquerait les manières de faire. Chacun composerait à sa façon le "menu » d'une cérémonie dont l'organisation lui appartiendrait en propre, parce que les obsèques reviendraient "de droit " à ceux qui sont concernés. Plus que l'interprétation savante de textes obscurs, c'est l'expression des émotions qui donneraient au rassemblement des proches la signification la plus juste d'une ultime relation. La messe sera dite. Mais le rituel signifiant aura presque lieu à l'insu du célébrant. On veut lire une lettre, dire un dernier mot, faire entendre de la musique, participer, même discrètement. Toutefois demandons-nous s'il s'agit bien de "nouveaux rituels " ? Ou posons cette autre question : la ritualité aurait-elle disparu, parce que la ritualisation (quand l'émotion partagée prévaut sur l'observance de règles extérieures) prendrait le pas sur les exégèses?

9 Voir Jean-Didier Urbain L'Archipel des morts, Paris, Payot, 1999. 
Lorsqu'on prétend qu'à l'autorité du "social " succèderait l'autonomie de "l'individu ", on oppose moins une thèse à une autre qu'on ne reproduit de l'une à l'autre un schéma explicatif identique. Au social déterminant de la sociologie classique, on propose une sociologie "nouvelle», mais qui reprend sans la penser davantage la notion d'individu qu'il fallait autrefois " dépasser ". Ainsi peut-on dire que si " au temps de Durkheim », le social déterminait l'individu, ce serait aujourd'hui l'individu qui déterminerait le social. Ces deux thèses, construites en miroir l'une de l'autre, sont tout également insuffisantes, et c'est avec l'illusion de "saisir un processus historique » que l'on croit pouvoir établir un passage entre une « époque » et une autre. Si le deuil est bien entendu social, ce n'est pas parce qu'il serait organisé par un opérateur magique - le "social »mais parce que la mort est cette question sans réponse à laquelle l'humanité s'affronte, ou encore parce qu'elle est cette limite qui détermine l'élaboration même de la culture. Dire que le deuil devient une affaire privée tient du commentaire de l'observable. Croire que "l'individu » doit gérer en dehors de tout rapport social la relation qu'il entretient avec les morts, n'a aucun sens.

Quand Sigmund Freud parle de travail du deuil ${ }^{10}$, c'est dans un tout autre sens que celui des sociologues qui font valoir les déterminations d'une société intégratrice et régulatrice. Ce qui est au travail, c'est le remaniement des investissements psychiques. Je ne pourrai prendre le temps de discuter ici d'une théorie que des psychanalystes eux-mêmes auront pu critiquer ${ }^{11}$. Ce qui me semble devoir être ici retenu, c'est la place qui se fait non pas à un individu qui aurait l'illusion de son autonomie, mais à un sujet divisé dont l'épreuve du deuil fait surgir la souffrance ${ }^{12}$. Le deuil n'est pas seulement peine et chagrin, colère et culpabilité, sentiments qui déstabiliseraient un individu qui "normalement " se maîtriserait lui-même. Il s'agit bien plus profondément de ce qui excède la capacité de contrôler ce qui arrive, c'est-à-dire de l'ouverture à l'inconnu et à l'intrigue ${ }^{13}$, dans la mesure où ce qui vient ne vient pas de soi. La mort, ici la distinction entre individu et société n'a décidément plus de sens, n'est pas une simple cessation des fonctions vitales. Elle est cette altérité qui atteste de toute son absence pourtant présente en chaque jour, de la non coïncidence qui affecte et le monde et l'individu. À cet endroit de la discussion, la sociologie durkheimienne ou sa version " contradictoire " (la sociologie " de l'individu ") sont totalement perdues : très exactement elles n'y comprennent plus rien. Elles n'ont pas de compétence pour comprendre le sens de la discussion.

Voyons par exemple ce dont il s'agit dans " le contrat obsèques ", vendu donc par les entreprises funéraires et les banques. On entend ainsi très simplement rappeler que cette nouveauté ne provient pas d'un mouvement social,

10 Voir Sigmund Freud, Métapsychologie, Paris, Gallimard, 1968, p. 150.

11 Voir Jean Allouch, Érotique du deuil au temps de la mort sèche, Paris, EPEL, 1997.

12 Voir Denis Vasse, Le Poids du réel, la souffrance, Paris, Seuil, 1983.

13 Voir Emmanuel Lévinas, Le Temps et l’autre, Paris, PUF, 1985. 
d'une invention d'acteurs. D'individus qui produiraient du «social », comme le croit la sociologie tourainienne. Ces entreprises vendent du produit financier. Beaucoup des vendeurs n'y croient guère eux-mêmes, mais la logique de l'entreprise suppose et impose qu'ils proposent à des clients ce que ceux-ci pourraient " choisir ». De quel choix s'agit-il ? Et qui concerne-t-il ?

Non pas des gens qui voudraient inventer leur propre rituel, mais de personnes inquiètes, en une période où les enfants sont au chômage, que ceux-ci ne puissent faire face à la dépense (de 3000 euros environ) pour payer les obsèques. Pour l'essentiel, le contrat obsèques ne consiste pas en un fantasme de régler ses propres funérailles, mais relève d'une incapacité familiale à faire face à une dépense d'argent. On veut moins prévoir la cérémonie (tout en régler) qu'une somme d'argent que les descendants pourraient avoir du mal à réunir. C'est dans un contexte de crise que le contrat obsèques peut avoir une signification objective. Inutile donc de décréter qu'une société s'individualise, ou qui gagnerait en autonomie pour chacun : c'est au contraire une dépendance qui est en cause. Que les industries du funéraire donnent de cette " réalité » une vision " positive ", ne doit pas étonner. Mais que la sociologie vienne à la remorque des faits observés, alors que ce n'est pas son métier, et qu'elle cautionne cette version $\mathrm{du}$ « mythe » de l'individualisation, cela n'est pas recevable.

La demande de contrat obsèques a bien peu à voir avec une autonomie de l'individu. Il s'agit d'une demande statistiquement faible : $10 \%$ des personnes seraient concernées en France. S’il faut considérer qu'une croissance devrait avoir lieu, reste que $80 \%$ des souscripteurs sont pour l'instant intéressés par ce que serait l'organisation financière de leurs propres funérailles, sans besoin donc de venir en parler avec une entreprise de pompes funèbres. Jean Ruellan, directeur de la communication chez PFG, nous renseigne. Pour « $38 \%$ des Français la raison de souscrire un contrat obsèques est d'enlever tout problème financier à ses proches. En 2010, ils sont $45 \%$ en moyenne et plus de $50 \%$ chez les ouvriers et ceux qui ont des enfants à charge $»^{14}$. Une évolution peut sans doute advenir. Jean Ruellan nous dit que « $81 \%$ [des Français] selon un sondage d'août 2010 se déclarent favorables à cette démarche ». Il nous dit aussi que " la deuxième raison majeure de souscrire un contrat de prévoyance est la volonté d'enlever des soucis d'organisation à ses proches, qui s'affirme elle aussi chez les cadres supérieurs et les habitants de la région parisienne ${ }^{15}$.

Au final, il semblerait que le service proposé par les entreprises de pompes funèbres fonctionne encore peu, dans la mesure où elles ne sauraient déterminer l'ensemble d'un cérémonial qui, en aucune culture, n'a jamais relevé de la seule volonté personnelle. Reste sans doute des évolutions possibles, mais sur

14 Jean Ruellan «Évolution des pratiques funéraires. La mort aujourd'hui », in Pratiques autour de la mort, enjeux acuméniques, sous la direction de Jacques-Noël Pérès, Paris, Desclée de Brouwer, 2012, p. 29. 
fond d'une part d'une fragilisation des liens de solidarités familiales ou d'une précarité économique, et d'autre part, sur fond d'une vision qui pourrait donner raison à la naïve sociologie de l'acteur, mais au risque de l'effondrement du soutènement symbolique de l'individu prétendument autonomisé.

Continuons avec la ritualité funéraire et son développement actuel, prenant forme de "personnalisation " (là où on devrait croire que l'individu décide ou devient " acteur »).

Ce n'est pas la "relation ", l'entente, la proximité qui fabriquent une société. Mais le rapport, la différenciation et la distanciation. Du côté de la relation, de l'entente et de la proximité, nous avons la ritualisation. Les obsèques seraient l'occasion d'une "dernière relation " avec le mort comme s'il fallait savoir tout de la perte que signifie sa "disparition ". Les funérailles seraient le moment où, au moins pour la dernière fois, l'on se tient en proximité du défunt. Selon la logique de cette ritualisation et au nom du bien qu'elle procurerait, il faut inciter les gens à prendre la parole, à devenir acteurs. L'implication des survivants définirait leur nouvelle responsabilité et leur capacité à une bonne prévoyance de leur économie psychique : la réussite des obsèques entraînerait probablement un " bon deuil ". Ainsi assiste-t-on aujourd'hui à des cérémonies parfois pénibles, et qui ne valent en aucune façon de "nouveaux rituels " comme certains semblent en décider trop vite. Il s'agirait, tout au contraire, de combler le vide de la ritualité, d'effacer l'impression de son indigence par un surcroît de mouvements, de paroles, d'interventions. Tout se passant ici comme si le silence était insupportable. Comme si ce qui se met en silence dans le travail d'une symbolisation, devait se mettre en signification au nom d'une positivisation des émotions ou des sentiments.

La ritualisation vient alors en lieu et place d'une ritualité dont on se défie, qu'on ne veut plus accueillir, parce qu'on se méfierait de laisser place aux ambiguïtés qu'elle comporte, notamment en faisant place au mort mais pour le congédier. L'important devient la signification positive, qui permettrait de " régler " au mieux les sentiments ou d'organiser un devoir de mémoire. La ritualité marque une transition et elle situe un seuil. Elle n'a pas pour fonction principale de clore de la meilleure façon une relation, mais d'inaugurer une autre mise en rapport avec le mort. Dans leur sagesse, les sociétés traditionnelles savaient bien la dangerosité d'un mort qui pourrait vouloir rester parmi les vivants, et la nécessité d'un accompagnement pour que ce mort accède, au terme d'un voyage, au rang de défunt. La ritualité n'a jamais rien réglé de manière définitive. Elle permet à la manière d'un cadre qui fait sa place à l'ambigu de se situer dans un dérèglement fondamental. Alors que sous couvert de "personnalisation ", c'est une proximité à l'endroit du mort qui se jouerait, au moment même où l'enjeu est à l'inverse celui d'une séparation et plus précisément celui d'une distanciation radicale ${ }^{16}$.

16 Voir Patrick Baudry (Armand Colin, 1989), La Place des morts, Paris, L'Harmattan, 2006. 
Une société n'est pas seulement forte parce qu'elle peut parler. Mais aussi et surtout, parce qu'elle peut faire de l'impossibilité de parler, le support d'un rapport à l'inconnu, à l'invisible, à l'altérité : c'est-à-dire à la mort. La symbolisation ne vient pas comme une réparatrice des manques ou des interstices. Elle ne sert pas à rafistoler le monde. Elle ne tient pas de la rustine ou de la colle. Elle n'agglutine pas les choses et les lieux. Elle ne cimente pas les hommes et les événements. Tout au contraire, elle fait place à l'impossibilité de savoir : en donnant à cette impossibilité non pas la marque d'une faute ou d'un manquement, mais une dimension d'ouverture vers ce qui nous lie invisiblement.

\section{Le symbolique, ou le dehors}

C'est à un remaniement des places dans une chaîne intergénérationnelle que la mort convoque. Le remaniement des places n'est pas qu'affaire de "succession ". Il s'agit toujours de savoir ce qui peut être fait de la place d'un autre qu'on ne saurait jamais " remplacer ". La transmission n'est pas qu'une convention ou un acte de générosité : «Laisser quelque chose à ceux qui suivent, passer le relais... ». Mais une obligation. Non pas une contrainte arbitraire mais la prise du sujet humain dans la construction intergénérationnelle. Prise de soi qui permet de trouver sa place. La transmission n'est pas seulement dans nos gènes. Elle relève aussi de notre gêne. D'un tourment ${ }^{17}$. La mort rappelle à ce qui ne vient pas de soi, à l'œuvre d'une transmission qui, tout en échappant à nos décisions, construit l'humanité d'un monde où vivre peut avoir du sens.

La société d'aujourd'hui tend à ne traiter de la mort que sur le versant d'une interaction avec le corps du mourant ${ }^{18}$ ou du décédé. Les spiritualismes d'illuminés qui se rencontrent aujourd'hui témoignent bien de la faillite qui menace la construction de l'espace des morts. On le voudrait contigu. En proximité. Or ce que nous enseignent les sociétés négro-africaines, c’est que la limite de la mort ne se trace pas par le fait même du décès. Il faut une société pour configurer un espace autre et qu'ainsi cette limite (qui n'existe pas immédiatement ou seulement concrètement), prenne forme d'un espace intermédiaire qui met en rapport avec l'invisible.

J'ai souvenir d'un voyage au Japon sur le mont Koya. Le lieu tient sa force notamment d'un cimetière, mais surtout de la présence d'un moine mort il y a fort longtemps, qui serait toutefois, sans qu'on puisse se l'expliquer bien, « encore là ». Sa tombe est présente. On peut la voir. Comme le moine protecteur serait encore ici, il existe au-delà de la tombe accessible, une autre tombe

17 Voir Pierre Legendre, L'Inestimable objet de la transmission, Paris, Fayard, 1985, p. 49.

18 Voir Patrick Baudry, Robert-William Higgins, Jacques Ricot, Le Mourant, Nantes, M-Editer, 2006. 
qui signifie sa mort et qui, celle-là, est inaccessible. On ne peut la voir que de loin. Â la manière d'un espace espacé : obligeant à la distance. Nous obligeant à la réserve. Cette tombe plus loin qu'on ne peut visiter, peut-on d'ailleurs vraiment la voir ? Ne figure-t-elle pas dans le registre du représentable, «l'irreprésentatibilité " du monde "plus loin ", pour après, sans nos compétences pour en savoir les périmètres, l'architecture ou les activités? Un monde autre. Mais qui fait de ce monde même, celui où nous vivons, où nous penserions avoir maîtrise de nos pas et de nos inventions, autre chose encore. En raison de la seconde tombe, la première tombe n'est plus seulement l'endroit où l'on dépose les morts. Elle est déjà l'espace énigmatique où la distance nous tient.

On peut avoir cette représentation que la distance sépare, qu'elle exclut et interdit. Or elle peut soutenir le sujet humain. Jean-Luc Marion le dit bien à propos d'un " trop de lumière ": "Écrasée d'un trop de lumière, la chose s'obscurcit d'autant. Non pas qu'elle disparaisse à la vue, mais parce que aucun monde ne l'accueille, et parce qu'elle n'en ménage aucun. ${ }^{19}$ Devant cette menace de réaliser un même monde où vivants et morts seraient co-présents, comment ne pas rappeler cette mise en garde : "Rien ne menace tant l'homme que de ne pas savoir en quel retrait il lui revient de demeurer. $»^{20}$ La force des sociétés dites traditionnelles (qui nous rappellent à nos propres questions) ne tient pas à leur connaissance des choses de l'au-delà, mais à leur possibilité de s'y reconnaître. Elle ne tient pas à leur discours, mais aux trous qui traversent la parole, et qui installent ce qui est hors sens dans la trame même de leur récit. Ce qui est " fabuleux ", ce n'est pas leurs " explications ", mais le sens d'une parole silencieuse qui préserve un écart. Ce n'est pas l'efficacité d'un arrangement qui est frappant. C'est presqu'à l'inverse une imperfection à laquelle il est fait place. En faisant place au négatif, ce qui s'accueille, ce n'est pas la mort, mais la vie.

Le cadavre situe la rupture irréversible de cette présence. C'est le mort qui part, dit-on. Ce sont surtout les survivants qui doivent s'absenter de celui qui, comme le disait Vladimir Jankélévitch ${ }^{21}$, n'est pas même absent. On comprend donc que les rites funéraires sont aussi bien et surtout institutionnels : en séparant la vie de la mort par la séparation du mort et des vivants, ils permettent l'association du défunt à la communauté symbolique de ceux qui lui survivent. En empêchant la folie - la confusion - la ritualité qui oblige à accompagner le mort jusqu’à la limite depuis laquelle se détermine la vie en commun, soutient la logique institutionnelle de la transmission et garantit la permanence d'une chaîne intergénérationnelle ${ }^{22}$. Quelles que soient les formes de leur mise en scène, les rites funéraires relèvent toujours d'une obligation

19 Jean-Luc Marion, L'Idole et la distance, Paris, Le Livre de Poche, 1991, p. 57-58.

20 Jean-Luc Marion, op. cit., p. 156.

21 Vladimir Jan kélévitch, La Mort, Paris, Flammarion, 1977.

22 Voir Pierre Legendre, Les Enfants du texte, Paris, Fayard, 1992. 
qui n'est pas seulement conventionnelle. C'est une opération symbolique qui s'y joue, dont la portée n'est pas limitable à leurs effets d'apaisement ou aux bénéfices de consolidations psychologiques qu'ils procurent. On ne ritualise pas les funérailles parce qu'il faut bien faire quelque chose, mais parce que faire quelque chose - ritualiser - est la seule réponse humaine à la question sans réponse de la mort.

Le " décédé » que produit la mort biologique, devient un mort que prend en charge la ritualité. Et l'enjeu de ce traitement est de construire autrement la place que le mort occupait dans l'espace des vivants : en tant que défunt. La retenue du mort prépare sa séparation, qui, elle-même, conditionne le remaniement des rapports entre vivants et les relations de ceux-ci en rapport de la place autre du défunt. La ritualité funéraire, quelles que soient ses formes de mises en scène, constitue fondamentalement une " défense culturelle " au sens où Georges Devereux employait cette expression ${ }^{23}$. Ce travail culturel que constitue le deuil - à la fois épreuve et soutien, affliction et "intelligence " de vie - est d'autant plus complexe et comprend d'autant plus d'enjeux sociaux en société négro-africaine que la personne qui meurt est bien une personne et non pas seulement un "individu». Une personne plurielle ${ }^{24}$, qui comprend toujours de l'autre (visible et invisible) en elle-même. Plusieurs représentations du corps, plusieurs âmes, plusieurs esprits, et plusieurs rapports aux morts et aux ancêtres... Le nom, le souffle, le double construisent ainsi une personnalité complexe et cohérente qui prend sens dans les réseaux de participations, de correspondances et d'oppositions où elle est située. Toutefois, nous autres modernes, sommes-nous si sûrs de notre stricte individualité ?

Louis-Vincent Thomas disait bien que notre humanité ne dispose que d'un stock limités d'attitudes ${ }^{25}$. Devant la mort, devant les morts, devant les malades mourants, devant et avec les familles en deuil, nous ne pouvons faire comme nous voudrions comme si chaque fois que la mort se produisait, nous pouvions inventer un protocole et croire à sa signification. Bien plus qu'une signification (l'interprétation simple d'un signe qu'on utiliserait), c'est un sens qui se trouve en jeu. Faire de l'individu cet acteur qui aurait pouvoir de produire des significations, c'est en fait l'assigner au devoir d'une interprétation étroite. C'est surtout méconnaître que l'individu échappe à la signification et l'obliger à un devoir social qui n'a rien à voir avec l'enjeu

23 Voir Georges Devereux, Essais d'ethnopsychiatrie générale, Paris, Gallimard, 1973, p. 8 : «Un stress est atypique si la culture ne dispose d'aucune défense préétablie, "produite en série", susceptible d'en atténuer ou d'en amortir le choc ".

24 Voir Louis-Vincent Thomas, "Le pluralisme cohérent de la notion de personne en Afrique Noire traditionnelle ", in La notion de personne en Afrique Noire, colloque international du CNRS, $n^{\circ}$ 544, Paris, 1971, republié dans Prétentaine, "Anthropologie de l'ailleurs - présence de Louis-Vincent Thomas ", IRSA, Montpellier, n 7/8, octobre 1997, p. 111-136. Voir Louis-Vincent Thomas, Rites de mort, Paris, Fayard, 1985. 
réel d'une ritualité funéraire. En fait de liberté, on le contraint à des choix préconstruits, quand ces choix n'ont rien à voir avec la responsabilité qui lui permet de trouver sa place. L'individu peut être cet homme ou cette femme qui font leur choix. Le sujet dont parle l'anthropologie ou la psychanalyse est par contre inséré dans une culture, inscrit en elle, dans le devoir de la transmette. Ce ne sont plus ici des conventions ou des valeurs qui possèderaient un caractère d'obligation arbitraire. Ce sont des principes de la vie en commun qui s'imposent radicalement.

Quand on dit que les rituels évoluent, il faut se demander à quel niveau ils se modifient. La ritualité conjugue l'intimité du sentiment avec la coutume sociale, peut-on dire. Mais cette liaison que l'on pourrait constater est très précisément ce qui fait l'enjeu de la ritualité. Son sens n'est pas d'accorder l'individu à la société. D'adapter cet individu à des normes nouvelles. Et la question n'est pas de savoir, si cet individu n'est pas d'autant plus adaptable à ces normes qu'il en serait lui-même le producteur. Si l'on prend la ritualité funéraire (c'est-à-dire la question de la mort) au sérieux, on ne peut se contenter de décrire des «transformations ». Il faut analyser ce qui dans lesdites transformations se perpétue ou se maltraite d'un enjeu de sens avec lequel il est malaisé de plaisanter. La ritualité intervient au-delà d'une société qui agirait sans les individus, et de ces individus eux-mêmes qui ne peuvent être réduits à leur propre individualité. Pour le dire autrement, la ritualité ne tient pas d'une emprise totalisante qui effacerait la singularité. Elle saisit le sujet comme " un parmi d'autres " pour reprendre le titre d'un ouvrage de Denis Vasse ${ }^{26}$. La ritualité nous fait partager, au risque de notre parole, non pas ce que nous aurions de commun, mais ce que nous avons en commun. Par tant d'aspects donc, mais jamais exposés, précisés ou explicités, la ritualité nous oblige à une responsabilité : conduire le mort jusque vers un dehors, en faisant de ce dehors ce qui modifie le rapport que nous avons à une vie, qui n'est pas tout entière notre "dedans ", pris que nous sommes par l'altérité, dans ce monde humain, d'une mort qui arrive.

Voici, pour conclure une " observation participante ». En voiture, on est passé à côté d'une montagne de pneus crevés. On a longé des carcasses de voitures rouillées. La maison du crématorium était au fond de l'impasse. La porte que j'ai ouverte m'a donné à voir le décor d'un café. Et je me suis demandé, en repoussant cette porte qui ne pouvait se fermer sans que celle du local technique s'entrouvre, si je ne m'étais pas trompé d'adresse.

J'avais cru que la serveuse du bar était là pour proposer un café. De fait elle le proposait. Puis elle est passée devant le zinc, et elle a rassemblé ses grands bras pour faire claquer ses mains. Elle a dit qu'il fallait avoir un moment de recueillement. Ce moment devait être professionnellement minuté dans sa 
cervelle. Assez vite, un autre grand mouvement de bras, une claque retentissante, et elle explique qu'on peut se rapprocher de l'écran vidéo (c'est très moderne). On y aperçoit un bonhomme qui s'est revêtu d'un chapeau qui serait cérémonial : un haut un peu chinois. C'est d'ailleurs en joignant ses deux mains jusque sous son menton, qu'il incline son tronc au moment où le cercueil entre dans le four. À la fin, la "serveuse » nous rappelle qu'il ne faut pas s'attarder. Elle explique qu'on peut emporter toutes les fleurs. Et qu'elle attend un autre " corps ".

\author{
Patrick Baudry \\ Université Bordeaux Montaigne \\ Patrick.Baudry@u-bordeaux-montaigne.fr
}

\title{
Résumé
}

Espace des morts, le cimetière est le lieu d'une mémoire et d'une ritualité qui affrontent les vivants à un dehors. Que devient cet espace, quand dans l'optique d'une ritualisation efficace, la cérémonie privilégie la relation entre les vivants ? Cet article met en question ce qui, sous formes d'une privatisation et d'une personnalisation, peut générer une désymbolisation.

\section{Mots-clés}

Mort, cimetière, ritualité, deuil, mémoire.

\section{Abstract \\ Place of the dead, the cemetery is the site of a memory and a rituality that confronts the living with an outside. What becomes of this space, when in the perspective of an efficient ritualization, ceremonies privilege the relationships between the living? This article that which, through the bias of privatization and personalization, may generate a desymoblization.}

\section{Keywords}

Death, cemetery, rituality, mourning, memory. 